\title{
Signal detection indices in schizophrenics on a visual, auditory, and bimodal Continuous Performance Test*
}

\author{
Lutz Mussgay and Ralph Hertwig \\ Department of Evaluative Psychiatry, Central Institute of Mental Health, Mannheim, F.R.G.
}

(Received 13 October 1989, accepted 13 May 1990)

\begin{abstract}
Signal detection indices (perceptual sensitivity) were calculated to compare performance of 24 male schizophrenic inpatients and 24 controls ( 12 alcoholics and 12 normals) on 4 different CPT-tests. A standard version (St) employed 1 target $(P=0.166)$ and 5 nontargets. In condition $\mathrm{V}$ stimuli were presented visually, in condition A acoustically and in condition VA bimodally ( 1 target $(P=0.333)$ and 1 nontarget). Compared to controls schizophrenics exhibited lower levels of perceptual sensitivity in all 4 conditions. They were especially impaired when stimuli were presented either acoustically or when they had to monitor 2 modalities simultaneously. Perceptual sensitivity of schizophrenics was sigificantly lower in conditions V, A, and VA than in condition St. For controls only condition VA led to lower values. Because St was always presented first, the possible explanation that vigilance decrement over time is responsible for the lowered perceptual sensitivity had to be ruled out. It could be shown that schizophrenics did not differ in sensitivity between conditions being later in task sequence. Controls, however, showed a slight decrement over time. Thus our finding should to a large extent be attributed to different task requirements. Response criterion beta yielded inconsistent results.
\end{abstract}

Key words: Signal detection theory; Continuous Performance Test; (Schizophrenia)

\section{INTRODUCTION}

An attention deficit in schizophrenic patients has been a central topic of research and theoretical reasoning (e.g. Nuechterlein, 1977; Garmezy, 1978; Nuechterlein and Dawson, 1984) since Kracpelin (1913) and Bleuler (1911) postulated disturbed attention processes characterising the schizophrenic disorder. Since then different experimental procedures have been used to provoke the supposed deficit in schizophrenic's attention. One of

Correspondence to: L. Mussgay, Research Psychologist, Department of Evaluative Psychiatry, Central Institute of Mental Health, D-6800 Mannheim 1, F.R.G.

*The data presented have been extracted from the first author's doctoral dissertation conducted at the laboratory of Prof. Dr. R. Cohen at the University of Konstanz, F.R.G. them is the Continuous Performance Test (CPT) of sustained attention. First described by Rosvold et al. (1956) the CPT requires the subject to press a button whenever the predesigned target stimulus occurs within the random sequence of 5-10 nontarget stimuli. Exposure times typically vary betwecn 40 and $200 \mathrm{~ms}$, interstimulus intervals between 1 and $1.5 \mathrm{~s}$. The test has been used in a variety of populations including hyperactive and learning-disabled children (e.g. Sostek et al., 1980; Swanson, 1981; Nuechterlein and Parasuraman, 1983; O’Dougherty et al., 1984) and high-risk children (e.g. Rutschmann et al., 1977; ErlenmeyerKimling and Cornblatt, 1978; MacCrimmon et al., 1980; Friedman et al., 1982; Grunebaum and Cohler, 1983; Nuechterlein and Parasuraman, 1983). In schizophrenic samples the CPT has been employed to study the neurophysiological causes of performance impairments (Orzack and Kornetsky, 1966) 
and to assess drug effects in schizophrenic patients (Kornetsky and Mirsky, 1966; Orzack et al., 1967; Kornetsky, 1972; Ericson et al., 1984). In studies by Asarnow and MacCrimmon (1978), Wohlberg and Kornetsky (1973), and Walker (1981) both hospitalized and remitted schizophrenic patients have been found to show significant deficits on the CPT when performance was evaluated by the two traditional parameters, i.e. crrors of omission, expressing that the subject failed to respond to a critical stimulus and errors of commission indicating that the subject erroneously responded to the occurrence of a non-critical stimulus. More recently different authors recurred to signal detection theory (SDT, Tanner and Swets, 1954) by calculating SDT-indices, such as $d^{\prime}$ and beta to separate the two hypothesized underlying processes of signal detection not being apparent in the raw crror scores (Rutschman et al., 1977; Nuechterlein and Parasuraman, 1983; Dougherty et al., 1984; Nuechterlein et al., 1986). The sensitivity measure $d^{\prime}$ refers to the sensory aspect of signal detection, i.e. the accuracy of target (signal) and nontarget (noise) discrimination. The response criterion beta corresponds to the amount of perceptual evidence that a subject demands before deciding that a given stimulus is a signal. Hence beta reflects cognitive nonsensory factors ('attitudes') and their influence on the process of signal detection (Davies and Parasuraman, 1982). SDT postulates that the response criterion dimension is independent of the sensitivity dimension.

Conceiving attention as a limited nonspecific capacity that can be allocated to specific processing demands, Nuechterlein and Dawson (1984) and Nuechterlein et al. (1986) conclude that the findings of impaired vigilance performance among schizophrenics and populations at risk for schizophrenia appear to depend on the processing load of the task. CPT versions that ask for a higher processing load by enhancing memory load, stimulus encoding, feature extraction, and response selection allow the differentiation of schizophrenics and children at risk from normal comparison subjects (Nuechterlein, 1985).

Within this area of research, the data of a project designed to examine evoked potentials and CPT performance of schizophrenics, alcoholics and normals (Mussgay, 1989) in CPT versions of differing processing demands offered a promising basis for the application of SDT. In this report we describe the results of four tasks selected from the ten of the original study.

In terms of SDT indices, it was expected (a) that schizophrenic subjects would show smaller values of perceptual sensitivity in comparison to control subjects. According to Beatty (1982) detection of auditory stimuli demand a higher processing load than detection of visual stimuli. On the basis of evoked potential data Duncan (1988) reports a modality-specific information processing impairment for schizophrenics that is stronger for the auditory than for the visual modality. Therefore it was (b) hypothesized that schizophrenic subjects would obtain smaller values of perceptual sensitivity in an auditory version of the CPT compared to the visual version. Finally (c) the presentation of a randomly alternating scquence of auditory and visual stimuli would further reduce the schizophrenics' sensitivity in comparison to the singlemodality presentations. For control subjects we did not expect any systematic variation of perceptual sensitivity across conditions.

No predictions were made for the response criterion beta because no explicit pay-off scheme was used and no feedback was given during the experiment.

\section{METHODS}

\section{Subjects}

Subjects were 24 male schizophrenic inpatients of the Psychiatrisches Landeskrankenhaus Reichenau meeting the criteria for a DSM-III diagnosis of schizophrenia (American Psychiatric Association, 1980) and 24 male control subjects composed of 12 alcoholic inpatients and 12 normal subjects. 19 schizophrenics were diagnosed paranoid-hallucinatory (ICD-9: 295.3), two patients were of the hebephrenic subtype (295.1), one patient was of the catatonic (295.2), one of the simplex (295.0) and one of the undifferentiated subtype (295.8). To avoid withdrawal effects alcoholics were not tested before 4 weeks after admission. Subjects with organic impairments were excluded. Normal control subjects were recruited from the staff of the hospital.

The age of the schizophrenics averaged 33.4 years, that of the controls 35.8 , ranging from 20 to 
53 and 23 to 55 , respectively with no significant difference ( $t$ test). In the mean schizophrenics had been ill for 10.3 years (range 2-34), their mean length of hospitalisation amounted to 49.6 months (range 5-228), their number of admissions to 6.2 (range 1-22). Twenty-two schizophrenics were on neuroleptic treatment, the dosage in equivalence to chlorpromazine (Davis, 1976) averaged $793 \mathrm{mg}$ (range 25-2812).

Mean educational training of schizophrenics amounted to 10.8 years, the vocational training to 1.5 years. Alcoholics were educated for 8.6 years, normals for 9.9 years. Vocational training averaged 2.4 years for alcoholics and 4.1 years for normals. All subjects were paid for taking part in the experiment.

\section{Apparatus and stimuli}

The visual letter stimuli produced by a Hewlett Packard 1350A Graphics Translator were presented on a green luminescent Hewlett Packard $1300 \mathrm{~A} \mathrm{X}-\mathrm{Y}$ display located in front of the subject at a distance of 70 inches within an area of $7.5 \times 7.5 \mathrm{~cm}$ formed by a grey frame. Auditory stimuli consisted of synthesized vowels (duration $195 \mathrm{~ms}$, loudness $80 \mathrm{db}$ ). They were reproduced from a magnetic tape by a Revox A-77 tape recorder and presented via headphones. The whole experiment was controlled by a Hewlett Packard 1000 laboratory computer in connection with a Hewlett Packard 2240 Measurement Controller.

\section{Procedure}

Data acquisition was completed in one session. After the subject was seated in a reclining chair in a sound attenuating chamber the electrodes for the recording of the electroencephalographic activity (FEG) were attached. For all subjects testing began with the standard version (condition $\mathrm{St}$ ) designed to replicate the reported performance deficits of adult schizophrenics on a CPT version that was typical of previous research (Kornetsky, 1972; Wohlberg and Kornetsky, 1973; Asarnow and MacCrimmon, 1978; Kornetsky and Orzack. 1978). The standard version St employed 5 nontarget letters (B, G, N, L, O) and the target letter (X). Stimulus duration was $100 \mathrm{~ms}$, the interstimulus interval lasted for $1200 \mathrm{~ms}$. The probability of the randomly occurring target stimulus was 0.166 . After a general introduction into the experimental setting subjects were instructed to press a handheld microswitch push button as fast as possible whenever the target ' $\mathrm{X}$ ' appeared on the screen. Of the standard version 180 practice trials in two blocks of 90 trials were given first to familiarize the subject with the general setup of the tasks to come and to allow for habituation of the perceptual system. Following condition St nine different versions of the CPT were administered in a randomized sequence.

For reasons of comparability it was necessary to restrict analysis to three conditions (i.e. V, A, and VA). For them task requirements were identical. Each condition used only one target and one nontarget, the probability for targets being 0.33 . In contrast, four of the remaining six conditions not considered here had been included in the study to test the effects of different numbers of targets and nontargets on task performance. In the remaining two conditions subjects had to ignore differences in the presentation of stimuli (right or left ear in an auditory version, capital vs. small letters in a visual task version). Thus the three conditions selected comprised tasks using either visual stimuli (V), auditory stimuli (A), or stimuli of both modalities (VA) in a randomly alternating sequence. To perform adequately subjects had to disregard stimulus modality. In contrast to condition St stimuli were the vowels $E, I, O$ and $U$. Across subjects the stimulus vowels were selected according to a latin square, but remained constant for both modalities. For condition VA the two vowels not being employed in conditions $V$ and $A$ were selected. Due to the requirements of an auditory presentation, stimulus duration had to be $195 \mathrm{~ms}$ in both modalities. The interstimulus interval was $1332 \mathrm{~ms}$. Each condition comprised 12 practice and 168 experimental trials, 56 heing targets. Each condition lasted 5 min. The whole session including one or more short breaks according to patients needs covered about $60-70 \mathrm{~min}$.

\section{RESULTS}

Sensitivity in condition $V, A$, and $V A$

After determination of conditional probabilities for hits and false alarms calculation of perceptual sensitivity followed standard procedures (Green and Swets, 1966). Both a parametric $d^{\prime}$, and a 
TABLE 1

Mean, standard deviation, and range of hit, false alarm rate, and nonparametric $P(A)$ in schizophrenics and controls

\begin{tabular}{|c|c|c|c|c|c|c|c|}
\hline & \multirow[t]{2}{*}{ Condition } & \multicolumn{3}{|c|}{ Schizophrenics } & \multicolumn{3}{|c|}{ Controls } \\
\hline & & Mean & $S D$ & Range & Mean & $S D$ & Range \\
\hline \multirow{4}{*}{$\begin{array}{l}\text { Hit } \\
\text { rate }\end{array}$} & $\mathrm{St}$ & 0.981 & 0.040 & $0.821-1.0$ & 0.996 & 0.010 & $0.964-1.0$ \\
\hline & V & 0.972 & 0.044 & $0.839-1.0$ & 0.992 & 0.019 & $0.911-1.0$ \\
\hline & $\Lambda$ & 0.950 & 0.077 & $0.714-1.0$ & 0.993 & 0.011 & $0.964-1.0$ \\
\hline & VA & 0.953 & 0.071 & $0.732-1.0$ & 0.994 & 0.008 & $0.982-1.0$ \\
\hline \multirow{4}{*}{$\begin{array}{l}\text { False } \\
\text { alarm } \\
\text { rate }\end{array}$} & St & 0.009 & 0.005 & $0.0-0.021$ & 0.007 & 0.003 & $0.0-0.014$ \\
\hline & V & 0.013 & 0.012 & $0.0-0.054$ & 0.010 & 0.007 & $0.0-0.027$ \\
\hline & $\mathrm{A}$ & 0.021 & 0.018 & $0.0-0.080$ & 0.008 & 0.005 & $0.0-0.027$ \\
\hline & $\mathrm{VA}$ & 0.033 & 0.020 & $0.0-0.080$ & 0.015 & 0.015 & $0.0-0.071$ \\
\hline \multirow[t]{4}{*}{$\mathrm{P}(\mathrm{A})$} & $\mathrm{St}$ & 0.994 & 0.011 & $0.95-1.0$ & 0.998 & 0.003 & $0.99-1.0$ \\
\hline & V & 0.990 & 0.014 & $0.95-1.0$ & 0.996 & 0.007 & $0.97-1.0$ \\
\hline & A & 0.982 & 0.023 & $0.92-1.0$ & 0.998 & 0.004 & $0.99-1.0$ \\
\hline & $\mathrm{VA}$ & 0.979 & 0.024 & $0.91-1.0$ & 0.996 & 0.005 & $0.98-1.0$ \\
\hline
\end{tabular}

nonparametric index $\mathrm{P}(\mathrm{A})$ were calculated (Grier, 1971; Davies and Parasuraman, 1982). (see Table 1 for conditional probabilities and the nonparametric $d^{\prime}$ ). In cases with a perfect hit or false alarm score for the estimation of $d^{\prime}$ a constant correction as suggested by Davies and Parasuraman (1982) was applied. Due to the extremely low rates of misses and false alarms in the alcoholic and normal subject samples only the nonparametric index will be reported as it makes no assumption about the underlying signal and noise distributions. $\mathrm{P}(\mathrm{A})$ correlated $r=0.94$ with $d^{\prime}$.

Fig. 1 presents the mean values of nonparametric sensitivity $\mathrm{P}(\mathrm{A})$ for each of the four conditions in schizophrenics and controls. The alcoholic and the normal subjects were combined into one control group after $t$ tests had established that the two groups did not differ significantly in $\mathrm{P}(\mathrm{A})$ on any of the four conditions. Statistical data analysis was done with SPSS-X. The data were analyzed by a 2 (groups) $\times 3$ (conditions) analysis of variance with groups (schizophrenics, controls) as a between subjects factor and conditions (visual, auditory, and auditory and visual modality) as a within subject factor. Condition St was treated separately because it differed in relevant task parameters (exposure time, number of nontargets, target probability, interstimulus interval) from the other conditions. The main effect of groups $(F(1,46)=11.00, P=0.002)$, the main effect of conditions $(F(2,92)=5.35, P=0.006)$, and the group $\times$

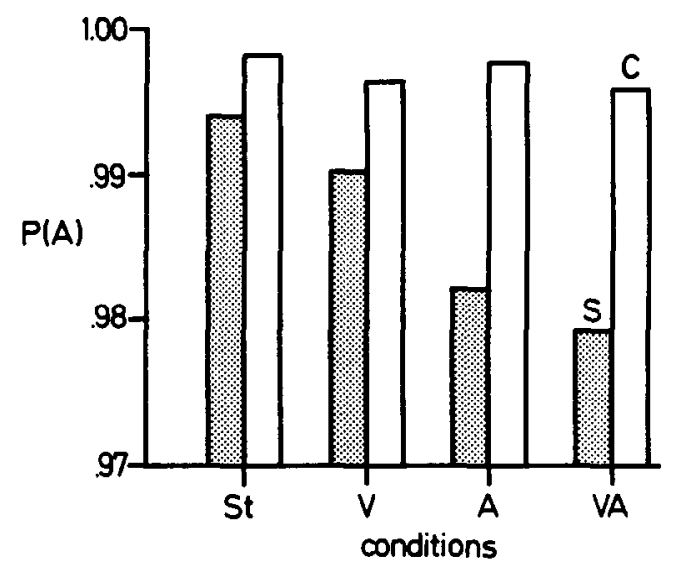

Fig. J. Nonparametric $\mathrm{P}(\mathrm{A})$ across conditions. $\mathrm{S}=$ schizophrenics; $\mathrm{C}=$ controls.

conditions interaction $(F(2,92)=4.97, P=0.009)$ reached statistical significance. Within-group posttests ( $t$ test for pooled variances) revealed that the schizophrenics' sensitivity in conditions A and VA was significantly lower than in condition $\mathrm{V}$ (conditions V-A: $\quad t(23)=2.47, \quad P=0.01 ; \quad$ V-VA: $t(23)=3.85, P=0.001)$ whereas conditions $\mathrm{A}$ and VA did not differ significantly $(t(23)=0.72$, NS), The control group did not differ in $\mathrm{P}(\mathrm{A})$ across conditions (V-A: $t(23)=-1.21, \quad \mathrm{NS} ; \mathrm{A}-\mathrm{VA}$ : $t(23)=1.71$, NS; V-VA: $t(23)=0.53$, NS). Betweengroup post-tests ( $t$ test for separate variances) revealed that schizophrenics obtained a significantly lower $\mathrm{P}(\mathrm{A})$ than controls in all conditions (V: 
$t(33.39)=-1.95, \quad P=0.03 ; \quad$ A: $\quad t(24.44)=-3.28$, $P=0.002 ;$ VA: $t(24.92)=-3.28, P=0.002)$.

Sensitivity in condition $S t$

The separately treated standard version $S t$ was compared in $\mathrm{P}(\mathrm{A})$ to conditions $\mathrm{V}, \mathrm{A}$, and VA by performing two-tailed $t$ tests for pooled variances. Schizophrenics attained significantly lower values in conditions $\mathrm{A}$ and VA compared to condition St. (St-V: $\quad t(23)=1.66, \quad \mathrm{NS} ; \quad$ St-A: $t(23)=2.95$, $P=0.007$; St-VA: $t(23)=4.04, P=0.001)$. The control subjects' sensitivity in St differed significantly only from condition VA (St-V: $t(23)=1.73$, NS; StA: $t(23)=1.08$, NS; St-VA: $t(23)=2.14, P=0.02$ ). A $t$ test comparison (separate variances, onetailed) of schizophrenics and controls sensitivity in condition St also yielded a significant difference $(t(26.52)=-1.76, P=0.04)$.

\section{Vigilance decrement over time}

Due to the fact that all subjects had to complete condition St first (with the remaining conditions following in a random sequence) the strong decline in perceptual sensitivity from condition St to conditions V, A, and VA in the schizophrenic sample may be produced by vigilance decrement over time instead of being an effect of more demanding task requirements. Such an explanation can be ruled out when the mean levels of sensitivity of conditions being later in sequence do not differ from those being presented earlier. Since the study originally was not designed to test vigilance decrement over time, we had to rearrange data to get a suitable, although not entirely satisfactory solution. Our four conditions allowed the separation of four time periods, the first always being made up of condition St. Whatever condition followed the presentation of St entered into the calculation of the mean levels of sensitivity of time period two. The same procedure applies for time periods three and four. So the mean level of each time period is formed of an equal proportion of conditions $\mathrm{V}, \mathrm{A}$, and VA. Unfortunately, sequence effects could not be considered. With the resulting sensitivity levels we performed a 2 (groups) $\times 4$ (time periods) analysis of variance with groups (schizophrenics, controls) as a between-subjects factor and time periods as a within-subject factor.

Fig. 2 presents mean perceptual sensitivity for each of the four time periods in schizophrenics and controls. The main effect of groups $(F(1,46)=$

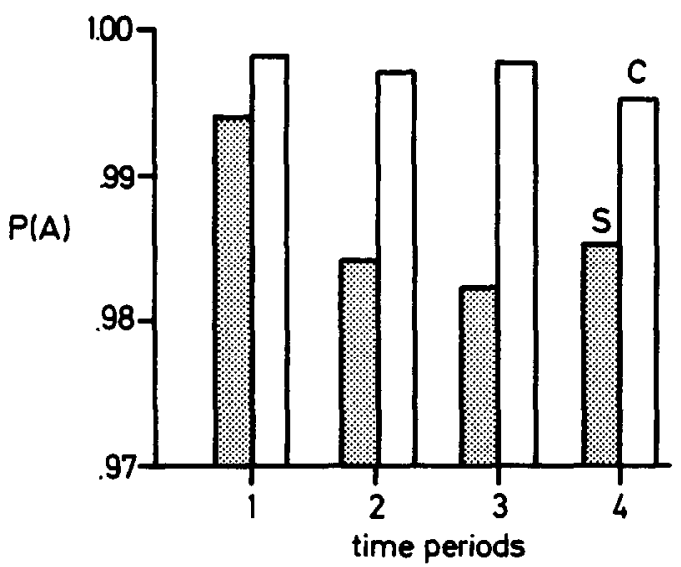

Fig. 2. Nonparametric $\mathrm{P}(\mathrm{A})$ at 4 subsequent time periods. $\mathrm{S}=$ schizophrenics, $\mathrm{C}=$ controls.

$10.33, P-0.002)$, the main effect of time periods $(F(3,138)=4.91, P=0.003)$, and the group $\times$ time periods interaction $(F(3,138)=3.14, P=0.027)$ attained statistical significance. Post-tests between groups (one-tailed $t$ tests, separate variances) indicated that schizophrenics differed significantly from controls in all of the four time periods (time period 1: $t(26.52)=-1.76, P=0.05 ; 2: t(24.65)=$ $-2.91, P=0.004 ; 3: t(24.44)=-3.28, P=0.002 ; 4$ : $t(28.98)=-2.29, \quad P=0.02)$. Within-group posttests (one-tailed $t$ test, pooled variances) revealed that in schizophrenics the decline in perceptual sensitivity reached statistical significance between time period 1 and time periods 2-4 (1-2: $t(23)=3.58, P=0.001 ; 1-3: t(23)=2.95, P=0.004$; $1-4: t(23)=2.37, P=0.01)$, whereas the differences within time periods 2,3 , and 4 did not attain statistical significance $(2-3: t(23)=0.52$, NS; $2-4$ : $t(23)=-0.29$, NS; $3-4: t(23)=-0.84$, NS). Obviously a vigilance decrement over time does not account for the sensitivity differences between conditions St and V, A, and VA in schizophrenics. For controls the differences in perceptual sensitivity did not reach statistical significance between time periods $1-2(t(23)=1.32, \mathrm{NS})$ and time periods $2-3$ $(/(23)=-0.60$, NS) but were significant between time periods 3 and $4(t(23)=-2.28, P=0.02)$ and periods 1 and $4(t(23)=2.50, \quad P=0.01)$ thus pointing to a slight decrease.

\section{Response criterion}

Two different indices of response criterion were calculated. The parametric index (Beta(Par)) uti- 
lized the ratio between the standardized ordinates of each individual's hit probability and false alarm probability (Hochhaus, 1972). Furthermore a nonparametric index of response criterion (Beta(Nonpar)), as suggested by Grier (1971) was calculated. As Table 2 indicates, the results were quite inconsistent. Significant correlations with indices of perceptual sensitivity were found for several conditions at that. This is in clear contradiction to signal detection theory which assumes independence. We therefore renounced analysing the response criterion.

\section{Correlations}

For schizophrenics, the nonparametric index of sensitivity $\mathrm{P}(\mathrm{A})$ is not significantly related to either duration of illness, length of hospitalization, or number of admissions. It also fails to reach significance for the neuroleptic dosage (in equivalence to chlorpromazine). However, with coefficients (Pearson Correlations) of $r=-0.30$ for condition $\mathrm{V}$, -0.29 for $\mathrm{A}$, and -0.31 for VA a slight improvement of perceptual sensitivity with increasing neuroleptic dosage seems to prevail. Performance in condition St obviously is not affected by neuroleptics $(r=-0.03)$.

For educational and vocational training no substantial relations with perceptual sensitivity are found, neither for schizophrenics nor for controls. The same applies for the age of controls with coefficients ranging from $r=-0.24$ to 0.18 . Quite contrary to expectation the perceptual sensitivity of schizophrenics seems to get better with increasing age (St: $r=0.31 ; \mathrm{V}: r=0.26 ; \mathrm{A}: r=0.45$, $P<0.05$; VA: $r=0.37$ ).

\section{DISCUSSION}

Analysis of our data according to the signal detection theory suggests that schizophrenics have significant impairments in perceptual sensitivity compared to control subjects. The findings of a lower overall sensitivity level correspond to significant deficits of hospitalized and remitted schizophrenic patients on the CPT reported by Orzack and Kornetsky (1966), Wohlberg and Kornetsky (1973) and Asarnow and MacCrimmon (1978). However, the extent of the deficit in perceptual sensitivity differed between the CPT conditions employed in this study. Consistent with our hypothesis schizophrenics attained significantly lower values of perceptual sensitivity in the auditory CPT version compared to the visual CPT version. In line with Duncan (1988) schizophrenics seem to be especially impaired when they have to process acoustical material. Recurring to the notion of Nuechterlein and Dawson (1984) and Nuechterlein et al. (1986) this result suggests that auditory detection of CPT stimuli demands a higher level of capacity-loading processing than visual detection in schizophrenics. This finding cannot be explained by diffcrent task difficulties as controls do not differ in perceptual sensitivity across visual and auditory modality.

Partly confirming our hypothesis and consistent with the studies of Kristofferson (1967) and Broen and Nakamura (1972) schizophrenics seem to be especially impaired when relevant stimuli are presented in more than one modality. They show lower levels of perceptual sensitivity in a dual-

TABLE 2

Means, and standard deviations of parametric and nonparametric Beta in schizophrenics and controls

\begin{tabular}{|c|c|c|c|c|c|c|c|c|}
\hline \multirow[t]{3}{*}{ Condition } & \multicolumn{4}{|c|}{ Schizophrenics } & \multicolumn{4}{|c|}{ Controls } \\
\hline & \multicolumn{2}{|c|}{ Beta(Par) } & \multicolumn{2}{|c|}{ Beta(Nonpar) } & \multicolumn{2}{|c|}{ Beta(Par) } & \multicolumn{2}{|c|}{ Beta(Nonpar) } \\
\hline & Mean & $S D$ & Mean & $S D$ & Mean & $S D$ & Mean & $S D$ \\
\hline St & -0.77 & 1.41 & -0.32 & 0.59 & -1.26 & 0.84 & -0.54 & 0.36 \\
\hline $\mathrm{V}$ & -0.35 & 1.38 & -0.13 & 0.62 & -1.07 & 0.88 & -0.48 & 0.41 \\
\hline $\mathrm{A}$ & -0.24 & 1.34 & -0.05 & 0.57 & -1.00 & 1.07 & -0.41 & 0.48 \\
\hline VA & -0.66 & 1.17 & -0.24 & 0.50 & -1.22 & 1.07 & -0.50 & 0.42 \\
\hline
\end{tabular}


modality condition. However, the difference to unimodal conditions reaches significance only for the visual condition, not for the auditory one. This seems to be a consequence of the higher processing load imposed by auditory detection per se, making that condition similar to the dual-modality task. As expected the control subjects did not show significantly different levels of perceptual sensitivity in any of the 3 experimental task conditions, but they revealed slightly lower levels in the dualmodality task compared to condition St. Obviously even for nonschizophrenics the monitoring of two stimulus sources exerts a degrading effect on sensitivity.

Schizophrenics and controls reached their highest level of perceptual sensitivity in the standard version, although this task in comparison is characterised by more demanding parameters (i.e., shorter exposure time, five nontargets). Analysis of vigilance over time revealed that the level of perceptual sensitivity in schizophrenics does not decline as a function of time-course. Controls, however, give evidence of a slight decrement. Thus, the reported difference between the standard version St and conditions V, A, and VA must be attributed mainly to task-specific aspects. Upon the nature of the relevant task differences can only be speculated. The adoption of different processing strategies in response to the number of nontarget stimuli might offer an explanation. Confronted with five nontargets subjects might develop the strategy to attend exclusively to the target ' $\mathrm{X}$ ' for a response, and disregard the nontargets totally. Whereas the discrimination of one target contrasted with one nontarget might lead subjects to decide for every presentation whether it's a 'go' or 'no-go' situation. Due to the higher processing load required by the second strategy such an explanation would be in line with the assumption of impaired perceptual sensitivity mirroring increased processing load as it was put forward by Nuechterlein and Dawson (1984). This tendency could have been further enhanced by the fact that condition St required only half the number of responses compared with conditions V, A, and VA. Here again more responses would lead to an increase in processing demands.

Different indices of the response criterion beta yielded contradicting results and, contrary to theoretical assumptions, interdependence of perceptual sensitivity and response criterion. The latter find- ing was reported by Nuechterlein and Parasuraman (1983) as well and seems not to be uncommon in other laboratories as mentioned in Rutschmann et al. (1977). These results seem to reflect the problem, that 'compared to indices of detectability considerable less effort has been invested in the development of a reliable index of response bias' (see Davies and Parasuraman, 1982, p. 49).

\section{REFERENCES}

American Psychiatric Association (1980) Diagnostic and statistical manual of mental disorders III. Washington, DC.

Asarnow, R.F. and MacCrimmon, D.J. (1978) Residual performance deficit in clinically remitted schizophrenics: a marker of schizophrenia? J. Abnorm. Psychol. 87, 597-608.

Beatty, J. (1982) Task-evoked pupillary responses, processing load and the structure of processing resources. Psycholog. Bull. 91, 276-292.

Bleuler, E. (1911) Dementia Praecox oder Gruppe der Schizophrenien. F. Deuticke, Leipzig, Vienna.

Broen, Jr., W.E. and Nakamura, Ch.Y. (1972) Reduced range of sensory sensitivity in chronic nonparanoid schizophrenics. J. Abnorm. Psychol. 79, 106-111.

Davies, D.R. and Parasuraman, R. (1982) The Psychology of Vigilance. Academic Press, London.

Davis, J.M. (1976) Comparative doses and costs of antipsychotic medication. Arch. Gen. Psychiatry 33, 858-861.

Duncan, C.C. (1988) Event-related brain potentials: a window on information processing in schizophrenia. Schizophr. Bull. 14, 199-203.

Erickson, W.D., Yellin, A.M., Hopwood, J.H., Realmuto, G.M. and Greenberg, L.M. (1984) The effects of neuroleptics on attention in adolescent schizophrenics. Biol. Psychiatry $19,745-753$.

Erlenmeyer-Kimling, L. and Cornblatt, B. (1978) Attentional measures in a study of children at high-risk for schizophrenia. J. Psychiatr. Res. 14, 93-98.

Friedman, D., Vaughan, H.G. and Erlenmeyer-Kimling, L. (1982) Cognitive related brain potentials in children at risk for schizophrenia: preliminary findings and methodological considerations. Schizophr. Bull. 8, 514-531.

Garmezy, N. (1978) Attentional processes in adult schizophrenia and in children at risk. J. Psychiatr. Res. 14. 3-34.

Green. D.M. and Swets. J.A. (1966) Signal Detection Theory and Psychophysics. Wiley, New York.

Grier, J.B. (1971) Non-parametric indexes for sensitivity and bias: computing formulas. Psycholog. Bull. 79 , 424-429.

Grunebaum, H. and Cohler, B.J. (1983) Children of parents hospitalized for mental illness, I. Attentional and interactional studies. J. Child. Contemp. Soc. 15, 43-55.

Hochhaus, L. (1972) A table for the calculation of $d^{\prime}$ and $B$. Psycholog. Bull. 77, 375-376. 
Kornetsky, C. (1972) The use of a simple test of attention as a measure of drug effects in schizophrenic patients. Psychopharmacology. 24, 99-106.

Kornetsky, C. and Mirsky, A.P. (1966) On certain psychopharmacological and physiological differenecs between schizophrenic and normal persons. Psychopharmacology. 8. 309-318.

Kornetsky, C. and Orzack, M.H. (1978) Psychological and behavioural correlates of attention dysfunction in schizophrenic patients. J. Psychiatr. Res. 14, 69-79.

Kräpelin, E. (1913) Psychiatrie: Ein Lehrbuch für Studierende und Ärzte, Vol. 3 (Klinische Psychiatrie, II). Johann Ambrosius Barth, Leipzig.

Kristofferson, M.W. (1967) Shifting attention between modalities: a comparison of schizophrenics and normals. J. Abnorm. Psychol. 72, 388-394.

MacCrimmon, D.J., Cleghorn, J.M., Asarnow, R.F. and Steffy, R.A. (1980) Children at risk for schizophrenia: clinical and attentional characteristics. Arch. Gen. Psychiatry 37, $671-674$

Mussgay, I. (1989) Der Continuous Performance Test (CPT) und Evozierte Potentiale bej schizophrenen Patienten: Effekte von Reizgruppierung und bimodaler Darbietung. HartungGorre, Konstanz.

Nuechterlein, K.H. (1977) Reaction time and attention in schizophrenia: a critical evaluation of the data and theories. Schizophr. Bull. 3, 373-428.

Nuechterlein, K.H. (1985) Converging evidence for vigilance deficit as a vulnerability indicator for schizophrenic disorders. In: M. Alpert (Ed.), Controversies in Schizophrenia. Changes and Constances. Guilford Press, New York.

Nuechterlein, K.H. and Dawson, M.E. (1984) Information processing and attentional functioning in the developmental course of schizophrenic disorders. Schizophr. Bull. 10, 160-203.

Nuechterlein, K.H. and Parasuraman, R. (1983) Visual sus- tained attention: image degradation produces rapid sensitivity decrement over time. Science $220,327-329$,

Nuechterlein, K.H., Edell, S., Noris, M. and Dawson, E. (1986) Attention vulnerability indicators, thought disorder and negative symptoms. Schizophr. Bull. 12, 408-423.

O'Daugherty, M., Nuechterlein, K.H. and Drew, B. (1984) Hyperactive and hypoxic children: signal detection, sustained attention and behaviour. J. Abnorm. Psychol. 93. $178-191$.

Orzack, M.H. and Kornetsky, C. (1966) Attention dysfunction in chronic schizophrenia. Arch. Gen. Psychiatry 14, 323-326.

Orzack, M.H., Kornetsky, C. and Freeman, H. (1967) The effects of daily administration of carphenazine on attention in the schizophrenic patient. Psychopharmacology 11, 3l-38.

Rosvold, H.E., Mirsky, A.F., Sarason, J., Bransome, E.D. and Beck, L.H. (1956) A continuous performance test of brain damage. J. Consult. Psychol. 20, 343-350.

Rutschmann, J., Cornblatt, B. and Erlenmeyer-Kimling, L. (1977) Sustained attention in children at risk for schizophrenia: report on a continuous performance test. Arch. Gen. Psychiatry $34,571-575$

Sostek, A.J., Buchsbaum, M.S. and Rapaport, J.L. (1980) Effects of amphetamine on vigilance performance in normal and hyperactive children. J. Abnorm. Child Psychol. 8, $49 \mathrm{I}-500$.

Swanson, L. (1981) Vigilance deficit in learning disabled children: a signal detection analysis. J. Child Psychol. Psychiatry 22, 393-399.

Tanner, W.P. and Swets, J.A. (1954) A decision making theory of visual detection. Psychol. Rev. 61, 401-409.

Walker, E. (1981) Attention and neuromotor functions of schizophrenics, schizoaffectives and patients with other affective disorders. Arch. Gen. Psychiatry 38, 1355-1358.

Wohlberg, G.W. and Kornetsky, C. (1973) Sustained attention in remitted schizophrenics. Arch. Gen. Psychiatry 28, $533-537$. 